\title{
La acreditación en la Facultad de Ingeniería Industrial de la Universidad Nacional Mayor de San Marcos y su importancia
}

\begin{abstract}
RESUMEN
En este artículo, se realiza el estudio de la información histórica, sobre el proceso de acreditación seguido por el programa de Ingeniería Industrial de la Universidad Nacional Mayor de San Marcos (UNMSM), de los procesos de acreditación en los cuales ha participado el Consejo Nacional de Acreditación de Colombia, de la calidad científica y profesional de los pares académicos, así como de la rigurosidad y seriedad de sus evaluaciónes, para demostrar la importancia que tiene la acreditación del programa de Ingeniería Industrial de la UNMSM, dentro del ámbito universitario, y de la ingeniería.
\end{abstract}

Palabras clave: acreditación, par académico, calidad, factor, programa

The aCcreditation in the Faculty OF INDUSTRIAL ENGINEERING OF THE National Mayor University of SAN MARcos AND his IMPORTANCE

\section{ABSTRACT}

In this article, makes the study of historical information on the accreditation process followed by the Industrial Engineering program at the Universidad Nacional Mayor de San Marcos (San Marcos University) of the accreditation process which has participated in the National accreditation of Colombia, the scientific and professional academic peers, as well as the rigor and seriousness of their assessments, to demonstrate the importance of the accreditation program UNMSM Industrial Engineering within the university, and engineering.

Keywords: accreditation, academic couple, quality, factor, program

\section{INTRODUCCIÓN}

Ante la proliferación de la oferta educativa en la enseñanza universitaria, son varios los caminos que han emprendido las diferentes instituciones universitarias con la finalidad de diferenciarse unas de otras y presentarse cada una como la mejor en su género. Ante este escenario, la Facultad de Ingeniería Industrial (FII) de la Universidad Nacional Mayor de San Marcos (UNMSM) de Lima Perú, opta por la acreditación internacional. El presente artículo tiene el objetivo de demostrar la importancia y seriedad de la acreditación obtenida, con el convencimiento de que cualquier esfuerzo por mejorar la educación en el mundo debe ser bienvenido por mínimo que sea.

\section{ANTECEDENTES}

Con la incursión del sector privado en la enseñanza superior universitaria, han sido creadas una gran cantidad de universidades, es un fenómeno mundial. En el Perú no hemos estado ajenos a este fenómeno. En la década del 90 del siglo XX se dio la ley N. ${ }^{\circ} 26439$ del 20/01/95, creando el Consejo Nacional para la Autorización de Funcionamiento de Universidades - CONAFU, órgano que ha permitido la creación de una variedad de universidades, privadas en mayor número. En la actualidad existen en funcionamiento 36 universidades nacionales, 76 privadas, y otras 22 (4 públicas y 18 privadas) en proceso de implementación [1, 2 y 3]. Entre estas últimas tenemos a la Universidad Nacional Tecnológica de San Juan de Lurigancho creada en Lima, según ley N.$^{\circ} 29659$, publicada el 28/01/11, y en provincias la Universidad Nacional Autónoma de Tayacaja Daniel Hernández Morillo (en Huancavelica), creada según ley N. ${ }^{\circ} 29716$, publicada el 23/06/1 [12].

Ante esta proliferación de universidades, el reto de cada una de ellas es diferenciarse unas de otras, unas han decidido lanzarse a una intensa difusión de sus servicios, al-

1 Ingeniero Industrial. Docente Asociado. Facultad de Ingeniería Industrial UNMSM. E-mail: orojasla@hotmail.com

2 Magíster - Ingeniero Industrial. Profesor del Departamento de Ingeniería de Sistemas e Informática, UNMSM.

E-mail: cquispea@industrial.unmsm.pe

3 Ingeniero Industrial. Profesor del Departamento de Diseño y Tecnología Industrial, UNMSM.

E-mail: wcalsina@industrial.unmsm.pe 
gunas han perfeccionado sus procesos educativos obteniendo su certificado de calidad ISO, otras han apostado por la acreditación de sus carreras.

En el país, el gobierno a través de la ley N ${ }^{\circ} 27154$ del año 1999 establece la acreditación obligatoria de las carreras formativas de profesionales en medicina, creando la Comisión para la Acreditación de Facultades o Escuelas de Medicina Humana (CAFME), cuyas funciones eran las de elaborar las normas para establecer los estándares mínimos de acreditación, así como la administración del proceso, su supervisión y evaluación permanente. De igual forma legisla y establece la obligatoriedad de la acreditación para las carreras formativas de educación.

En el Perú el Sistema Nacional de Acreditación y Certificación (SINEACE) [14] es el conjunto de organismos, normas y procedimientos estructurados e integrados funcionalmente encargados de definir las normas y estándares de acreditación y certificación, a fin de asegurar los niveles básicos de calidad que deben brindar las instituciones educativas, comprendidas en la ley general de educación No 28044, del 28/07/03 [10].

El Consejo de Evaluación, Acreditación y Certificación de la Calidad de la Educación Superior Universitaria - CONEAU, es un Organismo descentralizado del Ministerio de Educación, fue creado con la finalidad de contribuir al mejoramiento de la educación universitaria, que tiene entre sus funciones la evaluación de la calidad, y la acreditación de carreras de pregrado y postgrado de instituciones universitarias.

Existiendo los organismos encargados de velar por la calidad educativa, un conjunto de programas, especialmente de universidades particulares, han apostado por la acreditación, es así que algunos de ellos ya se encuentran acreditados.

\section{LA ACREDITACIÓN EN LA UNIVERSIDAD NA- CIONAL MAYOR DE SAN MARCOS [10 Y 11]}

En la UNMSM, las autoridades no han estado ajenas a esta problemática, el 28/02/02 se crea la Oficina de Calidad Académica y Acreditación (OCAA) como unidad dependiente de la Oficina General de Planificación, a través de la cual se empiezan a implementar políticas de Gestión de Calidad de la Educación Superior Universitaria; el 19/07/03 se suprime esta oficina y se crea la Oficina Central de Calidad Académica y Acreditación (OCCAA) como órgano de línea del rectorado, encargado de proponer las políticas y estrategias de evaluación de la calidad académica y la gestión conducentes a la autoevaluación y acreditación, así como monitorear los procesos que permitan su desarrollo. Así mismo, se creó una Oficina de Calidad Académica y Acreditación (OCAA) en cada una de las 20 facultades y en la Escuela de Posgrado. Dichos cambios fueron refrendados con RR-03331-R-04 del 16/07/04.

El 4/12/09 la escuela de Odontología es acreditada internacionalmente, por la Red Internacional de Evaluadores - RIEV, por un periodo de tres años. El año 2010 fue denominado por las autoridades universitarias como el "Año de la Calidad Educativa"; en este año fueron acreditadas internacionalmente las escuelas de Contabilidad (el 15/01/10, por el Consejo de Acreditación de Ciencias Sociales, Contables y Administrativas en la Educación Superior de Latinoamérica-CACECA, por un periodo de 4 años); Ingeniería Industrial (el 29/07/10, por el Consejo Nacional de Acreditación de Colombia - CNA, por un periodo de 6 años) [15 y 17]. En el año 2011, el 11/03/11 la Universidad Nacional Mayor de San Marcos fue acreditada institucionalmente por la Red Internacional de Evaluadores - RIEV [16], por un periodo de 2 años; el 16/05/11 se acreditó el programa de Medicina Humana a través de la Red Internacional de Evaluadores - RIEV, por un periodo de tres años. La escuela de Educación se encuentra en proceso de acreditación ante el CONEAU.

Las escuelas de Obstetricia, Enfermería, Tecnología Médica, Nutrición, Farmacia y Bioquímica, Psicología y Trabajo Social terminaron sus procesos de Autoevaluación y se encuentran en preparación para la acreditación nacional ante el CONEAU. El resto de escuelas, se encuentran en proceso de autoevaluación, cada una de ellas con diferente avance, pero todas con el mismo objetivo, que la comunidad reconozca y esté enterada de la calidad académica con la que se enseña en cada una de las especialidades de la UNMSM.

\section{LA ACREDITACIÓN EN LA FACULTAD DE INGE- NIERÍA INDUSTRIAL}

En la Facultad de Ingeniería Industrial de la UNMSM, el 10/01/03, a través de la RD 006-FII-03 se designa al Jefe de la Oficina de Calidad Académica y Acreditación, resolución que fue ratificada con la RR 03787-R-04 del 17/08/04; a partir de entonces se inicia el proceso de autoevaluación, el cual permitió detectar las debilidades y fortalezas del programa. Habiendo superado muchas de las debilidades en junio del año 2009, ante la carencia de un modelo nacional que permitiera certificar y acreditar la calidad académica en ingeniería, se decidió emprender la acreditación internacional, a través del Consejo Nacional de Acreditación de Colombia (previa autorización del CONEAU). El Consejo 
Nacional de Acreditación (CNA) es un organismo público y sin fines de lucro, con amplia experiencia en acreditación de programas de enseñanza universitaria en Colombia. El equipo que llevó a cabo la Autoevaluación estuvo compuesto por 50 personas: 26 docentes, 14 trabajadores administrativos, y 10 alumnos, organizados en 8 subcomités cada uno de los cuales trabajó sobre un factor (Proyecto y Misión Institucional, Estudiantes, Profesores, Procesos Académicos, Bienestar Institucional, Organización-Administración-Gestión, Egresados e Impacto Sobre el Medio, Recursos Físicos); laborando sobre un total de 42 características y 426 indicadores. En este proceso se contó con la amplia participación de docentes, alumnos y trabajadores administrativos, los cuales participaron entusiastamente de diversas maneras en la autoevaluación: haciendo labores como parte de los subcomités, asistiendo a reuniones informativas, a talleres de capacitación, colaborando en la sensibilización y difusión del proceso y otras actividades relacionadas con la acreditación. Es importante hacer notar la participación masiva de los estudiantes en las actividades de la entrega oficial de la resolución de acreditación por la CNA de Colombia.

Para la evaluación externa, CNA envió pares académicos en dos oportunidades:

- Primera misión: 2, 3 y 4 de diciembre del 2009. Se encargó de verificar las condiciones iniciales para poder iniciar el proceso de acreditación internacional. Se caracterizó por el nivel profesional, científico y académico de cada uno de sus integrantes.

\section{Dr. Jaime Bernal Villegas}

http://www.britishcouncil.org/jaime_bernal_villegas_spanish.pdf. (Visitado el 27-05-11)

\section{Dr. Pedro Prieto Pulido}

http://www.mineducacion.gov.co/cvn/1665/articles-185585_archivo_pdfpedro.pdf. (Visitado el 27-05-11)

- Segunda misión: 3, 4 y 5 de junio del 2010. Se encargaron de verificar las condiciones académicas, de organización y de infraestructura, con las cuales la FII - UNMSM forma a los profesionales en Ingeniería Industrial.

Esta misión se caracterizó por el nivel profesional, académico, y científico de cada uno de sus integrantes en la especialidad de Ingeniería Industrial. Pues todos son ingenieros industriales, con grados de maestro y doctor:

Dra. Martha Cecilia Gómez Pinilla de García http://campus.univalle.edu.co/2007/marzo/edicion45/direccion.php. (Visitado el 27-05-11)

\section{Dr. Elver Alfonso Bermeo Muñoz}

http://www.unidadenlace.net/Articulos/lng. \%20 Elver\%20Alfonso\%20Bermeo/. (Visitado el 27-05-11)

http://www.cenicafe.org/listado_de_reconocidos_2004.pdf. (Visitado el 27-05-11)

\section{Dr. Luis Bullón Salazar}

http://www.up.edu.pe/carrera/ingempresarial/ Paginas/PLA/Detalle.aspx? IdElemento $=345$. (Visitado el 27-05-11)

El 24/12/10, el CONEAU publicó a través del diario el Peruano los "Estándares para la Acreditación de las Carreras Profesionales Universitarias de Ingeniería"; abriendo paso a la posibilidad de la acreditación nacional, lo que se convierte en un nuevo reto para la FII-UNMSM. El modelo para la acreditación de ingenierías del CONEAU considera un total de 98 estándares que permiten medir aspectos de la gestión académica, administrativa y de investigación agrupados en tres dimensiónes: Gestión de la carrera, formación profesional, servicios de apoyo para la formación profesional; y nueve factores: Planificación, organización, dirección y control; enseñanza-aprendizaje; investigación; extensión universitaria y proyección social; docentes; infraestructura y equipamiento; bienestar, recursos financieros, grupos de interés.

\section{CONOCIENDO AL CNA}

EI CNA es un organismo gubernamental de Colombia [5], creado por Ley No 30 de 1992, forma parte del Sistema Nacional de Acreditación, junto con el Consejo Nacional de Educación Superior- CESU, es de naturaleza estrictamente académica. El Sistema Nacional de Acreditación, fue creado con el objetivo fundamental de garantizar a la sociedad que las instituciones de educación superior y los programas académicos acreditados cumplen con los más altos requisitos de calidad y realizan sus propósitos y objetivos.

Desde enero del año 1998 a mayo del año 2011, CNA ha evaluado 1279 programas académicos, habiendo acreditado a 1101 programas, 178 programas no fueron acreditados, como se puede observar en el Cuadro 1. Es importante observar que no todos los programas evaluados por CNA son acreditados.

De los 1101 programas acreditados, 403 (36.6\%) corresponden al área de Ingeniería, Arquitectura, Urbanismo y afines, como se muestra en el Cuadro 
Cuadro 1. Evolución de la acreditación en CNA (De enero 1998- a mayo 2011) [4, 8 y 9 ]

\begin{tabular}{cccccc}
\hline Año & $\begin{array}{c}\text { Total } \\
\text { Evaluados }\end{array}$ & $\begin{array}{c}\text { Total } \\
\text { Acreditados }\end{array}$ & $\begin{array}{c}\text { Acreditación } \\
\text { Primera }\end{array}$ & $\begin{array}{c}\text { Re-Acredita- } \\
\text { ción }\end{array}$ & No Acreditados \\
\hline Total & 1279 & 1101 & 787 & 314 & 178 \\
\hline
\end{tabular}

Fuente: CNA Colombia.

Cuadro 2. Número de acreditaciónes por área del conocimiento (Hasta mayo 2011)

\begin{tabular}{lll}
\hline \multicolumn{1}{c|}{ ÁREA } & TOTAL \\
\hline Agronomía, Veterinaria y afines & & \\
\cline { 1 - 1 } Bellas Artes & 698 \\
\cline { 1 - 1 } Ciencias de la Educación & \\
\cline { 1 - 2 } Ciencias de la Salud & \\
\hline Ciencias Sociales, Derecho, Ciencias Políticas & \\
\hline Economía, Administración, Contaduría y Afines & 403 \\
\hline Humanidades y Ciencias Religiosas & 1101 \\
\hline Matemáticas Ciencias Naturales & \\
\hline Ingeniería, Arquitectura, Urbanismo y afines & \\
\hline TOTAL &
\end{tabular}

Fuente: CNA Colombia

2, siendo el área de Ingeniería donde el CNA tiene la mayor cantidad de programas acreditados, que lo convierte en una institución de garantía, dada la experiencia que tiene en acreditar de programas de ingeniería.

\section{EL CNA Y LA INGENIERÍA INDUSTRIAL}

Dentro del rubro de las Ingenierías, han sido acreditadas (algunas reacreditadas) 20 programas de Ingeniería Industrial de diferentes universidades, lo que demuestra la experiencia que tiene el CNA en acreditación de programas de Ingeniería Industrial. (Ver Cuadro 3).

\section{EL CNA Y LA EVALUACIÓN INTERNACIONAL [6]}

El Consejo Nacional de Acreditación ha tomado la decisión de someterse a un proceso de evaluación externa internacional con el propósito de contribuir a la garantía de calidad de la educación superior y ayudar en la consolidación de la cultura de la calidad.
En el marco de la internacionalización de las agencias de acreditación esta práctica de evaluación viene imponiéndose debido a las exigencias de la globalización de los sistemas de educación superior, que conllevan la necesidad de que las decisiones de acreditación que toman las agencias, tengan validez o aceptación internacional, ya que los mercados profesionales y las redes científicas en las que se insertan los egresados de los programas acreditados, crecientemente operan en la dimensión transnacional. Para lograr este propósito se están desarrollando sistemas internacionales (regionales o inter-regionales) de reconocimiento mutuo de los sistemas y agencias nacionales de acreditación, como un primer paso en la dirección del reconocimiento y homologación de títulos académicos.

Como parte del proceso de internacionalización CNA está realizando los siguientes procesos y acciones: 
Cuadro 3. Programas acreditaditados en ingenierìa industrial

\begin{tabular}{|c|c|c|}
\hline Institución Superior & Estado & $\begin{array}{c}\text { Vigencia } \\
\text { (años) }\end{array}$ \\
\hline Escuela Colombiana de Ingeniería Julio Garavito & Acreditado & 4 \\
\hline Fundación Universidad del Norte & Reacreditado & 8 \\
\hline Pontificia Universidad Javeriana de Bogota & Acreditado & 7 \\
\hline Universidad Autónoma de Occidente & Acreditado & 4 \\
\hline Universidad Católica de Colombia & Acreditado & 4 \\
\hline Universidad de Antioquia & Reacreditado & 4 \\
\hline Universidad de la Sabana & Acreditado & 4 \\
\hline Universidad Distrital Francisco José de Caldas & Reacreditado & 6 \\
\hline Universidad ICESI & Acreditado & 4 \\
\hline Universidad Industrial de Santander & Reacreditado & 4 \\
\hline Universidad Libre & Acreditado & 4 \\
\hline Universidad Nacional de Colombia (Medellín) & Acreditado & 6 \\
\hline Universidad Santiago de Cali & Acreditado & 4 \\
\hline Universidad Tecnológica de Bolívar & Reacreditado & 6 \\
\hline Universidad Tecnológica de Pereira & Reacreditado & 7 \\
\hline Escuela de Ingeniería de Antioquia & Reacreditado & 4 \\
\hline Universidad de Ibagué & Reacreditado & 6 \\
\hline Pontificia Universidad Javeriana de Cali & Reacreditado & 4 \\
\hline Escuela de Ingeniería de Antioquia & Reacreditada & 4 \\
\hline Universidad Nacional de Colombia (Manizales) & Acreditado & 6 \\
\hline
\end{tabular}

Fuente: CNA de Colombia

- Participación activa en los proyectos pilotos para la acreditación internacional (regional) de la Red Iberoamericana para la Acreditación de la Calidad de la Educación Superior (RIACES).

- Participación de Colombia en el MERCOSUR Educativo y del Consejo Nacional de Acreditación en la Red de Agencias Nacionales de Acreditación RANA, así como adhesión al Sistema ARCUSUR.

- Desarrollo de alianzas internacionales para apoyar el inicio de la nueva modalidad de acreditación de maestrías y doctorados, siendo actualmente miembro de la Red Iberoamericana para la Acreditación de la Calidad de la Educación Superior (RIACES).

- Proyección Internacional del CNA en acciones transnacionales de acreditación.

- Desarrollar procesos de evaluación externa internacional.

Sobre esta base, CNA acreditó en el Perú al programa de Estomatología de la Universidad Peruana Cayetano Heredia y al Programa de Ingeniería Industrial de la Universidad Nacional Mayor de San Marcos del Perú. El Programa de Psicología de la Pontificia Universidad Católica del Perú se encuentra en proceso de acreditación. 


\section{BENEFICIOS DE LA ACREDITACIÓN}

Toda organización que obtiene la acreditación ha pasado por un proceso de autoevaluación, identificando fortalezas y debilidades, ejecutando las mejoras respectivas.

En cuanto al programa de Ingeniería Industrial de la UNMSM, entre los beneficios producto de la acreditación se puede detallar:

- Para la comunidad: Esta cuenta con un centro de formación profesional competente, cuya calidad en la enseñanza y organizacional está garantizada.

- El Colegio de Ingenieros del Perú recibe como miembros a egresados de un centro de formación profesional con alta calidad académica.

- Producto del proceso de acreditación los trabajadores disponen de mejores ambientes de trabajo, apropiado clima laboral, respeto a sus derechos laborales, capacitación, servicios de salud y recreación, entre otros.

- Para los alumnos: Estos cuentan con mejores ambientes de estudio, profesores de buen nivel profesional y académico, actualización del plan de estudios, bibliotecas actualizadas, laboratorios adecuadamente implementados, programas de recreación y deportes.

- Los docentes cuentan con cómodos ambientes para atención a alumnos, para investigación, para reuniones; disponen de aulas y equipos apropiados para el dictado de clases. También tienen facilidades para capacitación, subvención en la adquisición de libros, adquisición de laptops; y programas de atención de salud, recreación y deportes; así como el programa de ayuda para la elaboración de libros.

\section{CONCLUSIONES}

Del análisis de la información mostrada se concluye que la acreditación obtenida por la Fll de la UNMSM es importante, pues cuenta con toda la seriedad, rigurosidad académica y normativa, otorgada por una entidad de prestigio y con amplia experiencia en acreditación universitaria en Colombia como es el CNA.

\section{REFERENCIAS BIBLIOGRÁFICAS}

[1] Adonde.com. Directorio universidades Perú. http://www.adonde.com/eduuni.htm. (Visitado el 26-10-10)
[2] Altillo.com.Universidades del Perú.http://www. altillo.com/universidades/universidades_peru. asp. (Visitado el 26-10-10)

[3] Asamblea Nacional de Rectores. Universidades en Bajo la Juridicción de la ANR-Perú.http:// sigu.anr.edu.pe/ (Visitado el 27-05-11)

[4] Consejo Nacional de Acreditación. Evolución de la acreditación en Colombia http://www.cna. gov.co/1741/article-186348.html (Visitado el 2510-10, el 27-05-11)

[5] Consejo Nacional de Acreditación. El Sistema Nacional de Acreditación de Colombia http:// www.cna.gov.co/1741/article-186365.html. (Visitado el 25-10-10)

[6] Consejo Nacional de Acreditación. Estrategia de Internacionalización del Consejo Nacional de Colombia (CNA): http://www.cna.gov.co/1741/ articles-186362_recurso_1.pdf. (Visitado el 2610-10)

[7] Consejo Nacional de Acreditación. El sistema de educación superior de Colombia. http://www. cna.gov.co/1741/article-187279.html. (Visitado el 25-10-10)

[8] Chaparro Fernando. Midiendo el Impacto de la Acreditación, Estudios en Desarrollo en Colombia, http:// www.cna.gov.co/1741/articles-186504 PresImpacto_SNA.pdf. (Visitado el 04-11-10).

[9] Ley del Sistema Nacional de Evaluación, Acreditación y Certificación de la Calidad Educativa. http://www.unife.edu.pe/evaluación/sineace/ley. pdf (Visitado el 29-10-10)

[10] Oficina Central de Calidad Académica y Acreditación UNMSM (2011). Acreditación Institucional Internacional UNMSM, Lima Perú.

[11] Oficina Central de Calidad Académica y Acreditación UNMSM, Avances de la acreditación. http://www.unmsm.edu.pe/occaa/eventos/ proacred.html (Visitado el 27-05-11)

[12] PienzaLivre.com. La universidad pública en la encrucijada. http://pienzalivre.blogspot.com/ (Visitado el 01-07-11)

[13] Red Iberoamericana para la Acreditación de la Calidad de la Educación Superior (RIACES). Evaluación externa internacional del CNA. http:// www.riaces.net/index.php/acerca-de-riacesique-es-riaces/miembrosgroup1/miembros/92colombia.html. (Visitado el 30-10-10).

[14] Reglamento de la ley del SINEACE. http://www. anr.edu.pe/calidad/index.php?option=com_con 
tent\&task=view\&id=30\&ítemid=45. (Visitado el 29-10-10).

[15] Revista San Marcos al día. N. ${ }^{\circ} 221$. Edición del 20-09 al 03/10/10
[16] UNMSM. Medicina Humana acreditada a nivel Internacional. http://www.unmsm.edu. pe/?id=1578 (Visitado el 27-05-11).

[17] Semanario Micro Finanzas Año 2, N. ${ }^{\circ}$ 58. Septiembre 2010. 